Math. Nachr. 220 (2000), $45-57$

\title{
Spaces of Whitney Functions with Basis
}

By Alexander P. Goncharov of Ankara

(Received February 5, 1998)

(Revised Version December 30, 1998)

\begin{abstract}
We construct a basis in the spaces of Whitney functions $\mathcal{E}(K)$ for two model cases, where $K \subset \mathbb{R}$ is a sequence of closed intervals tending to a point. In the proof we use a convolution property for the coefficients of scaling Chebyshev polynomials.
\end{abstract}

\section{Introduction}

The problem of the existence of bases in concrete spaces of functions is one of the most important parts of the structure theory of Fréchet spaces. It became more exciting after the Grothendieck problem was solved in the negative in [13], [2], [1], [8], [11]. Still there is no example of a concrete functional nuclear $F$-space without a basis. For a long time the space of $C^{\infty}$-functions on a sharp cusp has been considered as a candidate for this role ([2], see also [12]).

Here we give a construction of a basis in the space of Whitney functions $\mathcal{E}(K)$ for two model cases, where the compact $K \subset \mathbb{R}$ is a sequence of intervals tending to a point. The proof is based on a convolution property for the coefficients of scaling Chebyshev polynomials (Sect. 3). The method can be applied for the construction of a special basis in the space $C^{\infty}[0,1]$ and subsequently for the space of $C^{\infty}$-functions on a graduated sharp cusp ([5]). As a tool we use the Dynin-Mityagin criterion for the property of being a basis in a nuclear Fréchet space (T.1.1 below).

\section{Preliminaries}

Let $K=\{0\} \cup \bigcup_{k=1}^{\infty} I_{k}$, where $I_{k}=\left[a_{k}, b_{k}\right]=\left[x_{k}-\delta_{k}, x_{k}+\delta_{k}\right]$. Let $h_{k}=a_{k}-b_{k+1}$, $b_{1} \leq 1$. Suppose that $a_{k} \downarrow 0, h_{k} \downarrow 0$ and $\delta_{k} \downarrow 0$.

1991 Mathematics Subject Classification. Primary: 46E10; Secondary: 46A35.

Keywords and phrases. $C^{\infty}$-functions, bases, Chebyshev polynomials. 
The topology in the space $\mathcal{E}(K)$ of Whitney functions is defined by the norms

$$
\|f\|_{p}=|f|_{p}+\sup \left\{\frac{\left|\left(R_{y}^{p} f\right)^{(i)}(x)\right|}{|x-y|^{p-i}}: x, y \in K, x \neq y, i=0,1, \ldots, p\right\},
$$

$p \in \mathbb{N}_{0}$, where $|f|_{p}=\sup \left\{\left|f^{(i)}(x)\right|: x \in K, i \leq p\right\}$ and $R_{y}^{p} f(x)=f(x)-T_{y}^{p} f(x)$ is the Taylor remainder. Let $\mathcal{E}_{0}(K)$ denote the subspace of $\mathcal{E}(K)$ consisting of functions which vanish at zero together with all their derivatives.

We will use the Chebyshev polynomials

$$
T_{N}(x)=\cos (N \cdot \arccos x)=\sum_{s=0}^{N} A_{s}^{(N)} \cdot x^{s},
$$

where for $s=N-2 j$

$$
A_{s}^{(N)}=(-1)^{j} N 2^{N-2 j-1} \frac{(N-j-1) !}{j !(N-2 j) !}, \quad j=0,1, \ldots,[N / 2]
$$

(see for instance [14], 6.10.6) and $A_{s}^{(N)}=0$ if one of the numbers $N, s$ is even and the other one is odd.

On the other hand, $\cos ^{p} t=\sum_{n=0}^{p} B_{n}^{(p)} \cos n t$, where $0 \leq B_{n}^{(p)} \leq 1$.

For $|\Delta| \geq 1$, and $0<\varepsilon \leq 1$ we have that

$$
T_{N}(\Delta+\varepsilon \cos t)=\sum_{s=0}^{N} A_{s}^{(N)} \sum_{p=0}^{s}\left(\begin{array}{l}
s \\
p
\end{array}\right) \Delta^{s-p} \varepsilon^{p} \cos ^{p} t=\sum_{n=0}^{N} \beta_{n}^{(N)}(\Delta, \varepsilon) \cos n t,
$$

where

$$
\beta_{n}^{(N)}(\Delta, \varepsilon)=\sum_{s=n}^{N} A_{s}^{(N)} \sum_{p=n}^{s} B_{n}^{(p)}\left(\begin{array}{l}
s \\
p
\end{array}\right) \Delta^{s-p} \varepsilon^{p}
$$

Since for $x \geq 1$

$$
\sum_{s=0}^{N}\left|A_{s}^{(N)}\right| x^{s}=\sum_{j=0}^{[N / 2]}(2 x)^{N-2 j} \frac{N}{2} \cdot \frac{(N-j-1) !}{j !(N-2 j) !}
$$

and $N / 2 \leq N-j$, we have

$$
\sum_{s=n}^{N}\left|A_{s}^{(N)}\right| x^{s} \leq \sum_{j=0}^{[N / 2]}\left(\begin{array}{c}
N-j \\
j
\end{array}\right)(2 x)^{N-2 j} \leq \sum_{i=0}^{N}\left(\begin{array}{c}
N \\
i
\end{array}\right)(2 x)^{N-i}=(2 x+1)^{N} .
$$

Therefore

$$
\left|\beta_{n}^{(N)}(\Delta, \varepsilon)\right| \leq \varepsilon^{n} \sum_{s=n}^{N}\left|A_{s}^{(N)}\right| \sum_{p=0}^{s}\left(\begin{array}{l}
s \\
p
\end{array}\right)\left|\Delta^{s-p}\right| \leq \varepsilon^{n}(2|\Delta|+3)^{N} .
$$

As Mityagin proved in [7] the Chebyshev polynomials give a basis in the space $C^{\infty}[-1,1]$ and this space is isomorphic to the space $s$ of rapidly decreasing sequences.

Let $T_{n k}$ denote the restriction to $I_{k}$ of the scaling Chebyshev polynomial, that is $T_{n k}(x)=T_{n}\left(\frac{x-x_{k}}{\delta_{k}}\right), x \in I_{k}$ and $T_{n k}=0$ for $x \in K \backslash I_{k}$. 
Let $\xi_{0 k}(f)=\frac{1}{\pi} \int_{0}^{\pi} f\left(x_{k}+\delta_{k} \cos t\right) d t, \xi_{n k}(f)=\frac{2}{\pi} \int_{0}^{\pi} f\left(x_{k}+\delta_{k} \cos t\right) \cos n t d t, n \in \mathbb{N}$. The functionals $\left(\xi_{n k}\right)$ are, clearly, biorthogonal to $\left(T_{n k}\right)$.

We will use the convention that $\sum_{i=m}^{n}=0$ for $m>n$ and $0^{0}=1$.

Since the space $\mathcal{E}(K)$ is nuclear, we can use the following criterion ([7], T.9 ).

Theorem 1.1. (Dynin-Mityagin.) Let $E$ be a nuclear Fréchet space and $\left\{e_{n} \in E, \eta_{n} \in E^{\prime}, n \in \mathbb{N}\right\}$ be a biorthogonal system such that the set of functionals $\left(\eta_{n}\right)_{n=1}^{\infty}$ is total over $E$. Let for every $p$ there exist $q$ and $C$ such that for all $n$

$$
\left\|e_{n}\right\|_{p} \cdot\left|\eta_{n}\right|_{-q} \leq C .
$$

Then the system $\left\{e_{n}, \eta_{n}\right\}$ is an absolute basis in $E$.

Here and subsequently, $|\cdot|_{-q}$ denotes the dual norm: for $\eta \in E^{\prime}$ let $|\eta|_{-q}=$ $\sup \left\{|\eta(f)|,\|f\|_{q} \leq 1\right\}$.

\section{Basis in the space $\mathcal{E}_{0}(K)$}

This section contains a slightly modified version of the joint result [3]. For the convenience of the reader we repeat it here, thus making the exposition self-contained. In an analogous way a basis was constructed in [6] for the subspace of the space of $C^{\infty}$-functions defined on a stepped sharp cusp, consisting of all the functions vanishing at the cusp.

For $f \in \mathcal{E}(K)$ let $f_{k}$ be equal to $f$ on $I_{k}$ and zero on $K \backslash I_{k}, X_{k}=\left\{f \in \mathcal{E}_{0}(K)\right.$ : $\left.\operatorname{supp} f \subset I_{k}\right\}$. Using Taylor expansion at zero of the corresponding extensions of the functions it is easy to obtain the following characterization for elements of the subspace $\mathcal{E}_{0}(K)$.

Lemma 2.1. The function $f$ from $\mathcal{E}(K)$ belongs to $\mathcal{E}_{0}(K)$ iff for every $r$ and for every $N$ there exists $C_{0}$ such that $\left|f_{k}\right|_{r} \leq C_{0} b_{k}^{N}\|f\|_{r+N}$ for any $k$.

Theorem 2.2. Let $K$ be a compact set as in the Preliminaries. If there exists $M$ such that $h_{k} \geq b_{k}^{M}$ for any $k$, then the system $\left\{T_{n k}, \xi_{n k}\right\}_{n=0, k=1}^{\infty, \infty}$ is a basis in the space $\mathcal{E}_{0}(K)$.

Proof. Clearly, the system of functionals $\xi_{n k}$ is total; thus we only need to check the condition (1.2). Fix $p \in \mathbb{N}_{0}$. Since $\left|T_{n}^{(j)}(x)\right| \leq n^{2 j}$ for $|x| \leq 1, j \leq n$, it follows that $\left|T_{n k}\right|_{p} \leq\left(n^{2} / \delta_{k}\right)^{u}$, where $u=\min \{n, p\}$. On the other hand, for any $f_{k} \in X_{k}$ we get $\left\|f_{k}\right\|_{p} \leq 4\left|f_{k}\right|_{p} h_{k}^{-p}$, as is easy to check. Thus $\left\|T_{n k}\right\|_{p} \leq 4 n^{2 u} \delta_{k}^{-u} h_{k}^{-p}$.

Let us evaluate the dual norms of the $\xi_{n k}$ as functionals on $C^{\infty}\left(I_{k}\right)$. Fix $r \in \mathbb{N}_{0}$, $f \in C^{\infty}\left(I_{k}\right)$. If $0<n \leq r$ then using the Taylor expansion of $f$ at $x_{k}$, we get

$$
\xi_{n k}(f)=\frac{2}{\pi} \int_{0}^{\pi} f^{(n)}(\theta) \frac{\delta_{k}^{n}}{n !} \cos ^{n} t \cdot \cos n t d t
$$


with $\theta=\theta(t) \in I_{k}$. Hence

$$
\left|\xi_{n k}(f)\right| \leq 2 \frac{\delta_{k}^{n}}{n !}|f|_{r} \leq C_{r}^{\prime}\left(\delta_{k} / n\right)^{n}|f|_{r} .
$$

For $n=0$ we replace the middle term in the last product by 1 and the bound is valid as well.

If $r<n$, then we can take the polynomial $Q_{n-1}$ of best approximation to $f$ on $I_{k}$ in the norm $|\cdot|_{0}$. Then by the Jackson theorem

$$
\left|\xi_{n k}(f)\right| \leq \frac{2}{\pi} \int_{0}^{\pi}\left|f-Q_{n-1}\right| d t \leq 2\left|f-Q_{n-1}\right|_{0} \leq C_{r}^{\prime \prime} \delta_{k}^{r} n^{-r}|f|_{r}
$$

(see for instance [10], 5.1.5). Thus with $C_{r}=\max \left\{C_{r}^{\prime}, C_{r}^{\prime \prime}\right\}, v=\min \{n, r\}$ we have

$$
\left|\xi_{n k}(f)\right| \leq C_{r}\left(\delta_{k} / n\right)^{v}|f|_{r} .
$$

Let now $r=2 p, q=(M+2) p, f \in \mathcal{E}_{0}(K)$. By the lemma,

Thus

$$
\left|\xi_{n k}(f)\right|=\left|\xi_{n k}\left(f_{k}\right)\right| \leq C_{r}\left(\delta_{k} / n\right)^{v} C_{0} b_{k}^{M p}\|f\|_{q} .
$$

and

$$
\left|\xi_{n k}\right|_{-q} \leq C_{r} C_{0}\left(\delta_{k} / n\right)^{v} h_{k}^{p}
$$

$$
\left\|T_{n k}\right\|_{p}\left|\xi_{n k}\right|_{-q} \leq 4 C_{r} C_{0} n^{2 u-v} \leq 4 C_{r} C_{0}(2 p)^{p} .
$$

This proves the theorem.

Corollary 2.3. $\mathcal{E}_{0}(K)=\left(\bigoplus X_{k}\right)_{s}$.

Here and in the sequel $X=\left(\bigoplus X_{k}\right)_{s}$ means that every $f \in X$ has a unique representation $f=\sum_{k=1}^{\infty} f_{k}$ with $f_{k} \in X_{k}$ and moreover for any $p \in \mathbb{N}$ the sequence $\left(\left\|f_{k}\right\|_{p}\right)_{k=1}^{\infty}$ is rapidly decreasing.

Proof. For any $p, Q \in \mathbb{N}$ let $N=M(Q+p), q=p+N$. As before,

$$
\left\|f_{k}\right\|_{p} \leq 4\left|f_{k}\right|_{p} h_{k}^{-p} \leq C_{q}\|f\|_{q} b_{k}^{N} h_{k}^{-p} \leq C_{q}\|f\|_{q} h_{k}^{Q} .
$$

Since $h_{k} \downarrow$ and $\sum h_{k}<\infty$, there exists a constant $C$ such that $h_{k} \leq C k^{-1}$. Thus $\left\|f_{k}\right\|_{p} \leq C_{q} C^{Q}\|f\|_{q} k^{-Q}$ and $\left(\left\|f_{k}\right\|_{p}\right) \in s$.

\section{Convolution property and a new biorthogonal system}

Fix in an arbitrary way three (maybe overlapping) finite intervals $I_{1}, I_{2}, I_{3} \subset \mathbb{R}$. Let $\widetilde{T_{n i}}, i=1,2,3, n \in \mathbb{N}_{0}$, be the corresponding scaling Chebyshev polynomials considered on $\mathbb{R}, p, r \in \mathbb{N}_{0}, p \leq r$. Then

$$
\sum_{q=p}^{r} \xi_{p 3}\left(\widetilde{T_{q 2}}\right) \xi_{q 2}\left(\widetilde{T_{r 1}}\right)=\xi_{p 3}\left(\widetilde{T_{r 1}}\right)
$$


This property is a corollary of the following fact from elementary linear algebra.

Lemma 3.1. Let $\left(e_{i 1}\right)_{i=1}^{n},\left(e_{i 2}\right)_{i=1}^{n},\left(e_{i 3}\right)_{i=1}^{n}$ be bases in an $n$-dimensional vector space, $\alpha_{i k}\left(e_{j l}\right)$ be the $i$-th coefficient in the expansion of $e_{j l}$ in the $k$-th basis. Then

$$
\sum_{q=1}^{n} \alpha_{p 3}\left(e_{q 2}\right) \alpha_{q 2}\left(e_{r 1}\right)=\alpha_{p 3}\left(e_{r 1}\right)
$$

Proof. The numbers $\alpha_{j 3}\left(e_{q 2}\right)$ form the transition matrix $M_{3 \leftarrow 2}$ from the second basis to the third. Here $j$ is the index of the row, $q$ is the index of the column. Analogously, $M_{2 \leftarrow 1}=\left[\alpha_{i 2}\left(e_{r 1}\right)\right]_{i, r=1}^{n, n}$. Thus in the sum above we see a product of the $p$-th row of $M_{3 \leftarrow 2}$ with the $r$-th column of $M_{2 \leftarrow 1}$, that is the $(p, r)$-th element of $M_{3 \leftarrow 2} M_{2 \leftarrow 1}=M_{3 \leftarrow 1}$.

Now if we apply this lemma to the Chebyshev bases (or arbitrary other scaling polynomials of increasing degree), then the terms with $q<p$ and $q>r$ vanish due to the orthogonality of $\xi_{q k}$ to all polynomials of degree less than $q$.

Fix a compact set $K$ as in the Preliminaries. For $n \in \mathbb{N}_{0}$ and $k \in \mathbb{N}$ we will denote by $P_{n k}$ the function equal to $\widetilde{T_{n k}}$ on $\left[0, b_{k}\right] \cap K$ and zero otherwise on $K$. Let $l: \mathbb{N} \rightarrow \mathbb{N}_{0}$ be a nondecreasing function. The concrete form of this function will depend on the compact set $K$ considered. We introduce a new biorthogonal system which will be a basis in $\mathcal{E}(K)$ for two model cases.

Fix a natural number $k$. If $n \geq l(k)$, then let $e_{n k}=T_{n k}, \eta_{n k}=\xi_{n k}$; for $n<l(k)$ let $e_{n k}=P_{n k}$,

$$
\eta_{n k}=\xi_{n k}-\sum_{i=n}^{l(k-1)-1} \xi_{n k}\left(P_{i k-1}\right) \xi_{i k-1}
$$

Lemma 3.2. The system of functionals $\left(\eta_{n k}\right)_{n=0, k=1}^{\infty, \infty}$ is total on $\mathcal{E}(K)$ and biorthogonal to $\left(e_{n k}\right)_{n=0, k=1}^{\infty, \infty}$.

Proof. Biorthogonality of $\{e, \eta\}$ can be easily checked from that of $\{T, \xi\}$ and from the convolution property.

Suppose that for some $f \in \mathcal{E}(K)$ we have $\eta_{n k}(f)=0$ for all $n, k$. Since $\eta_{n k}(f)=$ $\xi_{n k}(f)=\xi_{n k}\left(f_{k}\right)=0$ for $n \geq l(k)$, we see that $f_{k}$ is a polynomial on $I_{k}$ of degree at most $l(k)$. Now let us take $k_{0}=\min \{k: l(k)>0\}$. Then $\eta_{n k_{0}}=\xi_{n k_{0}}$ and $f_{k_{0}} \equiv 0$. Next for $k=k_{0}+1, n<l(k)$ we obtain $\xi_{n k}\left(f_{k}\right)=\eta_{n k}(f)=0$. Thus $f_{k} \equiv 0$. Continuing in this way we see that $f_{k} \equiv 0$ for any $k$ and $f \equiv 0$.

\section{Estimation of norms}

Let us first deduce some bounds for the norms of the elements and of the biorthogonal functionals for an arbitrary compact set $K$ of the above-mentioned type (we can omit here the condition of monotonicity of $\left.\left(h_{k}\right),\left(\delta_{k}\right)\right)$. 
Lemma 4.1. Let $d$ equal $b_{k-1}-x_{k}$ for $n<q<l(k)$ and $d=\delta_{k-1}$ for $q \leq n<l(k)$. Then for $n<l(k)$

$$
\left|\eta_{n k}\right|_{-q} \leq 4\left[\delta_{k}^{q}+d^{q} \sum_{i=n}^{l(k-1)-1}\left|\xi_{n k}\left(P_{i k-1}\right)\right|\right] .
$$

Proof. Let us remark that

$$
\eta_{n k}(f)=\frac{2}{\pi} \int_{0}^{\pi}\left[f\left(x_{k}+\delta_{k} \cos t\right) \cos n t-f\left(x_{k-1}+\delta_{k-1} \cos t\right) \sum_{i=n}^{l-1} \xi(P) \cos i t\right] d t .
$$

Here and in the sequel we use the notation

$$
\sum_{i=n}^{l-1} \xi(P):=\sum_{i=n}^{l(k-1)-1} \xi_{n k}\left(P_{i k-1}\right) .
$$

Suppose that $0<n<q<l(k)$. The case $n=0$ can be considered in the same manner with a change of the coefficient before the integral. Expanding both functions at $x_{k}$ up to the $q$-th degree, we represent the expression in the square brackets in the following form

$$
\begin{aligned}
{[\cdots]=} & \sum_{j=0}^{q-1} \frac{1}{j !} f^{(j)}\left(x_{k}\right) \delta_{k}^{j} \cos ^{j} t \cos n t \\
& -\sum_{j=0}^{q-1} \frac{1}{j !} f^{(j)}\left(x_{k}\right)\left(x_{k-1}-x_{k}+\delta_{k-1} \cos t\right)^{j} \sum_{i=n}^{l-1} \xi(P) \cos i t+\text { Remainder }
\end{aligned}
$$

which is equal to

$$
\begin{aligned}
\frac{1}{q !} f^{(q)}(\theta) \delta_{k}^{q} \cos ^{q} t \cos n t-\left[1 / q ! f^{(q)}\left(x_{k}\right)\left(x_{k-1}-x_{k}+\delta_{k-1} \cos t\right)^{q}\right. \\
\left.+R_{x_{k}}^{q} f\left(x_{k-1}+\delta_{k-1} \cos t\right)\right] \sum_{i=n}^{l-1} \xi(P) \cos i t
\end{aligned}
$$

with $\theta \in I_{k}$.

Let us show that the main part of the expansion will vanish after integration. By orthogonality we only need to consider the case $j \geq n$. We will compare the coefficients of $f^{(j)}\left(x_{k}\right) / j$ ! in both sums after integration. Since

$$
\frac{2}{\pi} \int_{0}^{\pi} \cos ^{j} t \cos n t d t=B_{n}^{(j)},
$$

the coefficient in the first sum equals $\delta_{k}^{j} B_{n}^{(j)}$.

For the second sum, due to the orthogonality, the corresponding coefficient is

$$
\sum_{m=n}^{j}\left(\begin{array}{c}
j \\
m
\end{array}\right)\left(x_{k-1}-x_{k}\right)^{j-m} \delta_{k-1}^{m}\left[\cos ^{m} t \sum_{i=n}^{m} \xi(P) \cos i t\right] .
$$

The expression in the square brackets after integration gives 


$$
\sum_{i=n}^{m} \xi(P) B_{i}^{(m)}
$$

Thus it remains to prove that

$$
\delta_{k}^{j} B_{n}^{(j)}=\sum_{m=n}^{j}\left(\begin{array}{c}
j \\
m
\end{array}\right)\left(x_{k-1}-x_{k}\right)^{j-m} \delta_{k-1}^{m} \sum_{i=n}^{m} \xi(P) B_{i}^{(m)} .
$$

Let us consider the sum (4.1) separately. Clearly,

$$
\xi_{n k}\left(P_{i k-1}\right)=\beta_{n}^{(i)}(\Delta, \varepsilon)=\sum_{s=n}^{i} A_{s}^{(i)} \sum_{p=n}^{s} B_{n}^{(p)}\left(\begin{array}{l}
s \\
p
\end{array}\right) \Delta^{s-p} \varepsilon^{p}
$$

with $\Delta=\frac{x_{k}-x_{k-1}}{\delta_{k-1}}, \varepsilon=\frac{\delta_{k}}{\delta_{k-1}}$. Changing the order of summation, we represent (4.1) as

$$
\sum_{s=n}^{m} \sum_{p=n}^{s} B_{n}^{(p)}\left(\begin{array}{l}
s \\
p
\end{array}\right) \Delta^{s-p} \varepsilon^{p} \sum_{i=s}^{m} B_{i}^{(m)} A_{s}^{(i)} .
$$

But the last sum here is the coefficient of $\cos ^{s} t$ in the expansion of $\cos ^{m} t$ in powers of $\cos t$, that is $\sum_{i=s}^{m} B_{i}^{(m)} A_{s}^{(i)}=1$ if $s=m$ and it is zero for $s<m$.

Hence

$$
\sum_{i=n}^{m} \xi(P) B_{i}^{(m)}=\sum_{p=n}^{m} B_{n}^{(p)}\left(\begin{array}{c}
m \\
p
\end{array}\right) \Delta^{m-p} \varepsilon^{p} .
$$

Therefore the right - hand side of (4.2) can be written as

$$
\begin{aligned}
& \sum_{m=n}^{j}\left(\begin{array}{c}
j \\
m
\end{array}\right)\left(-\Delta \delta_{k-1}\right)^{j-m} \delta_{k-1}^{m} \sum_{p=n}^{m} B_{n}^{(p)}\left(\begin{array}{c}
m \\
p
\end{array}\right) \Delta^{m-p} \varepsilon^{p} \\
= & \sum_{p=n}^{j} B_{n}^{(p)} \varepsilon^{p} \delta_{k-1}^{j} \Delta^{j-p} \sum_{m=p}^{j}\left(\begin{array}{c}
j \\
m
\end{array}\right)\left(\begin{array}{c}
m \\
p
\end{array}\right)(-1)^{j-m} .
\end{aligned}
$$

Since $\left(\begin{array}{c}j \\ m\end{array}\right)\left(\begin{array}{c}m \\ p\end{array}\right)=\left(\begin{array}{c}j \\ p\end{array}\right)\left(\begin{array}{c}j-p \\ m-p\end{array}\right)$ the last sum here is

$$
\left(\begin{array}{l}
j \\
p
\end{array}\right) \sum_{m=p}^{j}\left(\begin{array}{c}
j-p \\
m-p
\end{array}\right)(-1)^{j-m} 1^{m-p}=0
$$

for $p<j$. Thus we have only the case $p=j$ and the total sum is

$$
B_{n}^{(j)} \varepsilon^{j} \delta_{k-1}^{j}=B_{n}^{(j)} \delta_{k}^{j}
$$

and (4.2) is proved. Therefore,

$$
\begin{aligned}
\left|\eta_{n k}(f)\right| & \leq \frac{2}{\pi} \int_{0}^{\pi} \mid \text { Remainder } \mid d t \\
& \leq \frac{2}{q !}|f|_{q} \delta_{k}^{q}+\left(b_{k-1}-x_{k}\right)^{q}\left(\frac{2}{q !}|f|_{q}+2\|f\|_{q}\right) \sum_{i=n}^{l-1}|\xi(P)| .
\end{aligned}
$$

This establishes the formula for the first case. 
Let now $q \leq n<l(k)$. Then expanding up to degree $q$ the first function at $x_{k}$ and the second at $x_{k-1}$, we immediately have

$\eta_{n k}(f)=\frac{2}{\pi} \int_{0}^{\pi} f^{(q)}\left(\theta_{0}\right) \delta_{k}^{q} \cos ^{q} t \cos n t d t-\sum_{i=n}^{l-1} \xi(P) \cdot \frac{2}{\pi} \int_{0}^{\pi} f^{(q)}\left(\theta_{1}\right) \delta_{k-1}^{q} \cos ^{q} t \cos i t d t$

with $\theta_{j} \in I_{k-j}, j=0,1$. This proves the lemma.

We now turn to the elements $P_{n k}$. To simplify notation we write $R(f, p)$ instead of

$$
\sup \left\{\left|\left(R_{y}^{p} f\right)^{(i)}(x)\right| \cdot|x-y|^{i-p}, i \leq p, x, y \in K, x \neq y\right\} \text {. }
$$

Let as before denote $u=\min \{n, p\}$.

Lemma 4.2. The following estimates hold

$$
\left|P_{n k}\right|_{p} \leq 2^{n-1} \frac{n !}{(n-u) !} \delta_{k}^{-n} b_{k}^{n-u}, \quad R\left(P_{n k}, p\right) \leq e \cdot h_{k-1}^{-p}\left|P_{n k}\right|_{u}
$$

Proof. Let us write the function $P_{n k}$ in the form

$$
P_{n k}(x)=2^{n-1} \delta_{k}^{-n} \prod_{i=1}^{n}\left(x-\theta_{i}\right)
$$

where $\theta_{i} \in I_{k}, x \in\left[0, b_{k}\right] \cap K$. For $j \leq n$ the $j$-th derivative of $\prod_{i=1}^{n}\left(x-\theta_{i}\right)$ is a sum of $\frac{n !}{(n-j) !}$ terms of the type $\left(x-\theta_{i_{1}}\right) \cdots\left(x-\theta_{i_{n-j}}\right)$. Since $\left|x-\theta_{i}\right|<b_{k}$ for all $i$, we obtain the first bound of the lemma. Now if $x, y \in\left[0, b_{k}\right] \cap K$ and $p<n$, then $R\left(P_{n k}, p\right) \leq 2\left|P_{n k}\right|_{p}$ by the Lagrangian form for Taylor's remainder. If $p \geq n$, then it is zero. Suppose that $x$ and $y$ lie on different sides of the hole $h_{k-1}$. Let for instance $y \leq b_{k}$. Then $|x-y| \geq h_{k-1}$ and

$$
R\left(P_{n k}, p\right) \leq \sup _{i} \sum_{j=i}^{u} \frac{1}{(j-i) !} h_{k-1}^{j-p} \sup _{y}\left|P_{n k}^{(j)}(y)\right|
$$

and the second bound of the lemma is clear.

\section{Basis in $\mathcal{E}(\boldsymbol{K})$ for $\mathcal{E}(\boldsymbol{K}) \not 4 s$}

Here we consider a concrete compact set $K$. Let $\varphi: \mathbb{R}_{+} \rightarrow \mathbb{R}_{+}$be an arbitrary increasing function such that $\varphi(t) \geq t$, let $\delta_{k+1}=1 / \varphi\left(\delta_{k}^{-1}\right)$ and $I_{k}=\left[(b-2) \cdot \delta_{k}, b \cdot \delta_{k}\right]$.

If $t^{N} / \varphi(t) \rightarrow 0$ for all $N$ as $t \rightarrow \infty$, then the spaces $\mathcal{E}(K)$ and $s$ are not isomorphic (see T.3 in [4]). Moreover, one can easily construct a family, having the cardinality of the continuum, of pairwise nonisomorphic spaces $\mathcal{E}\left(K_{\alpha}\right)$ by choosing suitable scales of functions $\varphi_{\alpha}$ (see [4], T.1 for more details). 
Theorem 5.1. Suppose $b \geq 6$. Let the sequence $\left(\delta_{k}\right) \downarrow 0$ be such that $b \delta_{1} \leq 1$, $3 \delta_{k+1} \leq \delta_{k}$ for all $k$, and $\bar{K}=\{0\} \cup \bigcup_{k=1}^{\infty}\left[(b-2) \delta_{k}, b \delta_{k}\right]$. Then the system $\left\{e_{n k}, \eta_{n k}\right\}_{n=0, k=1}^{\infty, \infty}$ is a basis in the space $\mathcal{E}(K)$.

Proof. Here $h_{k}=(b-2) \delta_{k}-b \delta_{k+1}$. We check at once that $h_{k} \geq h_{k+1}$ and therefore the compact set $K$ satisfies the conditions of the Preliminaries. Moreover,

$$
h_{k} \geq 2 \delta_{k}, \quad h_{k} \geq b_{k+1}
$$

and we can apply [4] for the isomorphic classification of the space $\mathcal{E}(K)$.

Let us fix $l=l(k)$ such that

$$
(2 b+1)^{l(k)} \cdot \delta_{k-1} \leq 1 .
$$

In order to get this, one can take $l=[(k-2) \nu]$ with $\nu=\frac{\ln 3}{\ln (2 b+1)}$, because

$$
(2 b+1)^{l} \delta_{k-1} \leq(2 b+1)^{\nu(k-2)} 3^{-k+2} \delta_{1}=\delta_{1}<1 .
$$

In addition for this $l$ we can take $k_{0}$ such that

$$
l(k) \leq \delta_{k-1}^{-1}, \quad k>k_{0}
$$

Since $\xi_{n k}\left(P_{i k-1}\right)=\beta_{n}^{(i)}(\Delta, \varepsilon)$ with $|\Delta|=\left|\frac{x_{k}-x_{k-1}}{\delta_{k-1}}\right|<b-1, \varepsilon=\frac{\delta_{k}}{\delta_{k-1}}$ we conclude from (1.1) and (5.1) that

$$
\sum_{i=n}^{l-1}|\xi(P)| \leq \sum_{i=n}^{l(k-1)-1} \varepsilon^{n}(2 b+1)^{i} \leq \varepsilon^{n}(2 b+1)^{l(k)} \leq \delta_{k}^{n} \delta_{k-1}^{-n-1} .
$$

Fix $p \in \mathbb{N}, q=3 p+2$ and $k_{q}$ with $k_{q} \geq k_{0}, l\left(k_{q}\right) \geq q$. Let $C_{0}=\max \left\|e_{n k}\right\|_{p} \cdot\left|\eta_{n k}\right|_{-q}$ for $0 \leq n \leq q, 1 \leq k \leq k_{q}$.

If $k \leq k_{q}, n>q$ or $k>k_{q}$ and $n \geq l(k)$, then $e_{n k}=T_{n k}, \eta_{n k}=\xi_{n k}$ due to the choice of $l(k)$ and $k_{q}$. As in Theorem 2.2 we have the bound

$$
\left|T_{n k}\right|_{p} \leq n^{2 p} \cdot \delta_{k}^{-p}
$$

Arguing as in Lemma 4.2, we get for $y \in I_{k}, x \in K \backslash I_{k}$

$$
\sum_{j=i}^{p} \frac{1}{(j-i) !}\left|T_{n k}^{(j)}(y)\right||x-y|^{j-p} \leq \sum_{j=i}^{p} \frac{1}{(j-i) !} n^{2 j} \delta_{k}^{-j} h_{k}^{j-p} \leq e \cdot n^{2 p} \delta_{k}^{-p},
$$

as $\delta_{k} \leq h_{k}$. Thus $\left\|T_{n k}\right\|_{p} \leq 4 \cdot n^{2 p} \delta_{k}^{-p}$.

For biorthogonal functionals it is enough in this case to use (2.1) with $q$ instead of $r$

$$
\left|\xi_{n k}\right|_{-q} \leq C_{q}\left(\delta_{k} / n\right)^{q}
$$

as $n \geq q$. Therefore, $\left\|T_{n k}\right\|_{p} \cdot\left|\xi_{n k}\right|_{-q} \leq 4 C_{q}$.

It remains to analyze the case $k>k_{q}, 0 \leq n<l(k)$. Here $e_{n k}=P_{n k}, \eta_{n k}$ is defined by (3.1). Fix $k$. 
If $0 \leq n<p$, then by Lemma 4.2

$$
\left\|P_{n k}\right\|_{p} \leq C_{1}^{\prime} \delta_{k}^{-n} \delta_{k-1}^{-p},
$$

where $C_{1}^{\prime}$ does not depend on $k$ and $n$.

On the other hand, by Lemma 4.1 and (5.3)

$$
\left|\eta_{n k}\right|_{-q} \leq 4\left[\delta_{k}^{q}+\left(b \cdot \delta_{k-1}\right)^{q} \cdot \delta_{k}^{n} \delta_{k-1}^{-n-1}\right] .
$$

Therefore,

$$
\left\|P_{n k}\right\|_{p}\left|\eta_{n k}\right|_{-q} \leq 4 C_{1}^{\prime}\left[\delta_{k}^{q-n} \delta_{k-1}^{-p}+b^{q} \cdot \delta_{k-1}^{q-n-p-1}\right] \leq 4 C_{1}^{\prime}\left(1+b^{q}\right)=C_{1} .
$$

If $p \leq n<q$, then with the same bound for $\left|\eta_{n k}\right|_{-q}$ we have by Lemma 4.2

$$
\left|P_{n k}\right|_{p} \leq 2^{n-1} n^{p} \delta_{k}^{-n}\left(b \delta_{k}\right)^{n-p}, \quad R\left(P_{n k}, p\right) \leq e\left(2 \delta_{k-1}\right)^{-p}\left|P_{n k}\right|_{p} .
$$

Thus $\left\|P_{n k}\right\|_{p} \leq C_{2}^{\prime} \cdot \delta_{k}^{-p} \delta_{k-1}^{-p}$ and

$$
\left\|P_{n k}\right\|_{p}\left|\eta_{n k}\right|_{-q} \leq 4 C_{2}^{\prime}\left[\delta_{k}^{q-p} \delta_{k-1}^{-p}+b^{q}\left(\delta_{k} / \delta_{k-1}\right)^{n-p} \delta_{k-1}^{q-2 p-1}\right] \leq 4 C_{2}^{\prime}\left(1+b^{q}\right)=C_{2} .
$$

Suppose $q \leq n<l(k)$. Then

$$
\left|P_{n k}\right|_{p} \leq \frac{1}{2}(2 b)^{n} n^{p} \delta_{k}^{-p}, \quad R\left(P_{n k}, p\right) \leq e\left(2 \delta_{k-1}\right)^{-p}\left|P_{n k}\right|_{p} .
$$

Therefore,

$$
\left\|P_{n k}\right\|_{p} \leq 2(2 b)^{l} l^{p} \delta_{k}^{-p} \delta_{k-1}^{-p} \leq 2 \delta_{k}^{-p} \delta_{k-1}^{-2 p-1},
$$

by (5.1) and (5.2). Also, by Lemma 4.1 and (5.3)

and

$$
\left|\eta_{n k}\right|_{-q} \leq 4\left[\delta_{k}^{q}+\delta_{k-1}^{q} \cdot \delta_{k}^{n} \delta_{k-1}^{-n-1}\right]
$$

$$
\left\|P_{n k}\right\|_{p}\left|\eta_{n k}\right|_{-q} \leq 8\left[\delta_{k}^{q-p} \delta_{k-1}^{-2 p-1}+\left(\delta_{k} / \delta_{k-1}\right)^{n-p} \delta_{k-1}^{q-3 p-2}\right] \leq 8=C_{3},
$$

due to the choice of $q$. The constant $C=\max _{i \leq 3} C_{i}$ does not depend on $n, k$, hence the theorem follows from Theorem 1.1 and Lemma 3.2.

Now let us introduce the projections

$$
S_{k}=\sum_{j=1}^{k-1} \sum_{n=0}^{l(j)-1} \eta_{n j}(\cdot) P_{n j}, \quad Q_{k}=\sum_{n=0}^{\infty} \eta_{n k}(\cdot) e_{n k}
$$

in the space $\mathcal{E}(K)$. Clearly, $Q_{k}(f)=f-S_{k}(f)$ on $I_{k}, Q_{k}(f)$ is a polynomial of degree $l(k)-1$ on $\left[0, b_{k+1}\right] \cap K$ and $Q_{k}(f)=0$ otherwise on $K$. Let $X_{k}=Q_{k}(\mathcal{E}(K))$.

Corollary 5.2. $\mathcal{E}(K)=\left(\bigoplus X_{k}\right)_{s}$. 
Proof. In fact, it is enough to show that for all $p$ and for all $M$ there exist $q$ and $C$ such that

$$
\sum_{k=1}^{\infty}\left\|Q_{k}(f)\right\|_{p} \cdot k^{M} \leq C\|f\|_{q}
$$

We can repeat all the arguments of the theorem with $q=3(p+1)+M$ and show in this way that the double series

$$
\sum_{k=1}^{\infty} \sum_{n=0}^{\infty}\left|\eta_{n k}(f)\right| \cdot\left\|e_{n k}\right\|_{p} k^{M}
$$

is convergent.

\section{Basis in $\mathcal{E}(K)$ for $\mathcal{E}(K) \simeq s$}

Let now $b_{k}=2^{-k+1}=2 b_{k+1}, \delta_{k}=2^{-k-2}=2 \delta_{k+1}$ for $k \in \mathbb{N}$. Clearly, $a_{k}=$ $6 \delta_{k}, h_{k}=2 \delta_{k}$. From [9] and [4] it follows that the spaces $\mathcal{E}(K)$ and $s$ are isomorphic. Let us give an explicit form of the basis in $\mathcal{E}(K)$ which can be applied for the construction of a special basis in the space $C^{\infty}[0,1]$.

Theorem 6.1. Let $K=\{0\} \cup \bigcup_{k=1}^{\infty}\left[3 \cdot 2^{-k-1}, 2^{-k+1}\right]$. Then the system $\left\{e_{n k}, \eta_{n k}\right\}_{n=0, k=1}^{\infty, \infty}$ is a basis in the space $\mathcal{E}(K)$.

Proof. Let us take $l(k)=[k / 4]$. Since for our case $\Delta=-7 / 2, \varepsilon<1$, we replace (5.3) by

$$
\sum_{i=n}^{l-1}|\xi(P)| \leq \sum_{i=n}^{l(k-1)-1} 10^{i}<10^{l(k-1)}<\delta_{k}^{-1} .
$$

Fix $p \in \mathbb{N}$, take $q=3 p+2$ and $k_{q}=4 q$.

Let $C_{0}$ be the same as in Theorem 5.1. The estimates of $\left\|e_{n k}\right\|_{p} \cdot\left|\eta_{n k}\right|_{-q}$ for $n \geq l(k)$ or $n<l(k), k \leq k_{q}$ are the same as above. Similarly, for fixed $k>k_{q}$ if $n<p$ then

$$
\left|P_{n k}\right|_{p} \leq 2^{n-1} n ! \delta_{k}^{-n}, \quad R\left(P_{n k}, p\right) \leq e \cdot\left(4 \delta_{k}\right)^{-p}\left|P_{n k}\right|_{n} .
$$

Therefore

$$
\left\|P_{n k}\right\|_{p} \leq p ! \delta_{k}^{-2 p}
$$

By Lemma 4.1 and (6.1)

$$
\left|\eta_{n k}\right|_{-q} \leq 4\left[\delta_{k}^{q}+\left(b_{k-1}-x_{k}\right)^{q} \cdot \delta_{k}^{-1}\right] .
$$

Here $b_{k-1}-x_{k}=8 \delta_{k-1}-7 \delta_{k}=9 \delta_{k}$. Hence, $\left|\eta_{n k}\right|_{-q} \leq C \delta_{k}^{q-1}$, where $C$ does not depend on $n, k$, and the product $\left\|P_{n k}\right\|_{p} \cdot\left|\eta_{n k}\right|_{-q}$ is uniformly bounded.

If $p \leq n<q$, then $\left\|P_{n k}\right\|_{p} \leq 2^{4 q} q^{p} \delta_{k}^{-2 p}$, as is easy to check. For $\left|\eta_{n k}\right|_{-q}$ we use the previous bound and obtain the desired conclusion. 
Suppose now that $q \leq n$. Then by Lemma 4.2

$$
\left|P_{n k}\right|_{p} \leq 2^{n-1} n^{p} \delta_{k}^{-n}\left(8 \delta_{k}\right)^{n-p}
$$

and

$$
\left\|P_{n k}\right\|_{p} \leq(1+e)\left(4 \delta_{k}\right)^{-p}\left|P_{n k}\right|_{p} \leq 2^{4 n+1} n^{p} \delta_{k}^{-2 p} .
$$

Since $n<l(k) \leq k / 4$ and $k<\delta_{k}^{-1}$, we have

$$
2^{4 n+1} n^{p} \leq 2 \cdot 2^{k} k^{p}<\delta_{k}^{-p-1}
$$

Thus $\left\|P_{n k}\right\|_{p} \leq \delta_{k}^{-3 p-1}$.

From Lemma 4.1 with $\delta_{k-1}=2 \delta_{k}$ and (6.1) it follws that

$$
\left|\eta_{n k}\right|_{-q} \leq 4\left[\delta_{k}^{q}+\left(2 \delta_{k}\right)^{q} \cdot \delta_{k}^{-1}\right] \leq 2^{q+3} \delta_{k}^{q-1}
$$

Therefore $\left\|P_{n k}\right\|_{p} \cdot\left|\eta_{n k}\right|_{-q} \leq 2^{q+3}$ due to the choice of $q$.

Thus for the system $\left\{e_{n k}, \eta_{n k}\right\}_{n=0, k=1}^{\infty, \infty}$ we have the Dynin-Mityagin estimate (1.2) and in view of Lemma 3.2 the proof is complete.

In the same manner as above we can obtain the following

Corollary 6.2. $\mathcal{E}(K)=\left(\bigoplus X_{k}\right)_{s}$.

Remark 6.3. The basis in $\mathcal{E}(K)$ cannot be constructed as an extension of the basis in the subspace of the functions vanishing at zero. In fact, $\mathcal{E}_{0}(K)$ is not complemented in $\mathcal{E}(K)$ because the quotient space $\mathcal{E}(K) / \mathcal{E}_{0}(K)$ is isomorphic to the space $\omega=\mathbb{R}^{\mathbb{N}}$ and does not have a continuous norm.

In turn if we take the basis projection

$$
Q_{0}=\sum_{k=1}^{\infty} \sum_{n=l(k)}^{\infty} \xi_{n k}(\cdot) T_{n k}
$$

on the "vanishing part" $X_{0}=Q_{0}(\mathcal{E}(K))$ of the space $\mathcal{E}(K)$ with $X_{1}=\left(I-Q_{0}\right)(\mathcal{E}(K))$, then $X_{0} \subset \mathcal{E}_{0}(K)$ as a proper subspace and the exact sequence

$$
0 \longrightarrow X_{0} \longrightarrow \mathcal{E}(K) \longrightarrow X_{1} \longrightarrow 0
$$

splits.

\section{References}

[1] Bessaga, C.: A Nuclear Fréchet Space without Basis; Variation on a Theme of Djakov and Mityagin, Acad. Polon. Sci. Ser. Math., Astronom., Phis. 24, 7(1976), 471-473

[2] Djakov, P. B., and Mityagin, B. S.: Modified Construction of Nuclear Fréchet Spaces without Basis, J. Funct. Anal. 23 (1976), 415-433

[3] Goncharov, A.P., and Zahariuta, V.P.: On the Existence of Basis in Spaces of Whitney Functions on Special Compact Sets in $\mathbb{R}$, METU, Preprint Series 58 (1993), Ankara, Turkey 
[4] Goncharov, A., and Kocatepe, M.: Isomorphic Classification of the Spaces of Whitney Functions, Michigan Math. J. 44 (1997), 555-577

[5] Goncharov, A. P., and Zahariuta, V.P.: Basis in the Space of $C^{\infty}$-Functions on a Sharp Cusp, in preparation

[6] Kondakov, V. P., and Zahariuta, V. P.: On Bases in Spaces of Infinitely Differentiable Functions on Special Domains with Cusp, Note di Matematica XII (1992), 99-106

[7] Mityagin, B. S.: Approximate Dimension and Bases in Nuclear Spaces, Russian Math. Surveys 16 (1961), $59-127$

[8] Moscatelli, V. B.:, Fréchet Spaces without Continuous Norm and without Bases, Bull. London Math. Soc. 12 (1980), 63-66

[9] Tidten, M.: Kriterien für die Existenz von Ausdehnungsoperatoren zu $\mathcal{E}(K)$ für kompakte Teilmengen $K$ von $\mathbb{R}$, Arch. Math.(Basel) 40 (1983), 73-81

[10] Timan, A. F.: Theory of Approximation of Functions of a Real Variable, Pergamon Press, 1963

[11] Vogt, D.: An Example of a Nuclear Fréchet Space without the Bounded Approximation Property, Math. Z. 182 (1983), 265-267

[12] Zerner, M.: Développement en Séries de Polynômes Orthonormaux des Fonctions Indéfiniment Différentiables, C. R. Acad. Sci. Paris 268 (1969), 218-220

[13] Zobin, N. M., and Mityagin, B. S.: Examples of Nuclear Fréchet Spaces without Basis, Funct. Anal. i ego priloz. 84 (1974), 35-47 (Russian)

[14] Zwillinger, D.: Standard Mathematical Tables and Formulae, 30th edition, CRC Press, 1996

\author{
Department of Mathematics \\ Bilkent University \\ 06533 Ankara \\ Turkey \\ and \\ Department of Mathematics \\ Civil Building University \\ Rostov-na-Donu \\ Russia \\ E-mail: \\ goncha@fen.bilkent.edu.tr
}

